UT-Komaba 98-4

UTHEP-378

DPNU-98-10

\title{
Multicanonical simulation of 3D dynamical triangulation model and a new phase structure
}

\author{
Tomohiro Hotta \\ Institute of Physics, University of Tokyo, \\ Meguro-ku, Tokyo 153, Japan \\ Taku Izubuchi \\ Institute of Physics, University of Tsukuba, \\ Tsukuba, Ibaraki 305, Japan \\ and \\ Jun Nishimura \\ Department of Physics, Nagoya University, \\ Chikusa-ku, Nagoya 464-01, Japan
}

February, 1998

\begin{abstract}
We apply the multicanonical technique to the three dimensional dynamical triangulation model, which is known to exhibit a first order phase transition with the Einstein-Hilbert action. We first clarify the first order nature of the phase transition with the Einstein-Hilbert action in several ways including a high precision finite size scaling analysis. We then add a new local term to the action and confirm the conjecture made through the MCRG technique that the line of the first order phase transition extends to the expanded phase diagram, ending at a point. Fractal dimension at the end point is measured to be around three up to the present size.
\end{abstract}




\section{Introduction}

Quantum gravity is one of the most important topics in particle physics. The attempts to construct it within ordinary field theories confront a difficulty due to the fact that the theory is perturbatively unrenormalizable. This does not mean immediately that the attempts fail, since it might be possible to construct it nonperturbatively as is the case with the 3D nonlinear sigma model. The lattice regularization provides a natural approach in this context. In the case of quantum gravity, since the dynamical variable is given by the metric of the space time, how to discretize the space time is itself a nontrivial problem. Above all, it seems difficult to preserve the general coordinate invariance manifestly. The success of the dynamical triangulation model in two dimensions [1] summarized by its equivalence to the Liouville theory [i] in the continuum limit shows that the model provides a natural framework to study quantum gravity nonperturbatively within ordinary field theories. Although the restoration of the general coordinate invariance in the continuum limit is only supported by the success in two dimensions, we might consider that the summation over all the triangulation with the equal weight forms the basis of the restoration. The situation with the other lattice regularization of quantum gravity known as the Regge calculus is very obscure [3].

The above point of view motivated intensive studies of the dynamical triangulation model in higher dimensions through Monte Carlo simulation. It turned out, however, that with an action obtained by naive discretization of the Einstein-Hilbert action, the phase transition observed is of first order both in three dimensions [泪 and in four dimensions [5, 6]. The signal of the first order phase transition is much weaker in $4 \mathrm{D}$ than in $3 \mathrm{D}$. We note that this does not necessarily mean that it is easier to construct a continuum theory out of this model in 4D than in 3D, and we consider that this is merely related to the existence of singular vertices in the crumpled phase in $4 \mathrm{D},[7,8]$. By adding a local term which suppresses the singular vertices, we observe a first order phase transition in 4D, which is even stronger than in 3D. Indeed, from the viewpoint of perturbative unrenormalizability, the situation in 4D must be severer than in $3 \mathrm{D}$. The $\epsilon$-expansion of $(2+\epsilon)$-dimensional quantum gravity [9] suggests the existence of a non-trivial fixed point, and this result is of course more reliable for smaller $\epsilon$ naively. We therefore concentrate on 3D dynamical triangulation model in this paper. Three-dimensional quantum gravity is worth being studied in itself, since it can be also regarded as the 
first quantization of membranes, which is considered to be a fundamental object in the M-theory [10].

In lattice theories in general, it is often preferable (e.g. improved actions to reduce the finite lattice spacing effects) or even necessary (e.g. the Wilson term to eliminate fermion doublers) to modify the lattice actions from that obtained by naive discretization of the action in the continuum. We may therefore hope to obtain a second order phase transition in the present case by adding a local term to the naive Einstein-Hilbert action. Once the continuum limit can be taken, the continuum theory constructed is expected to be independent of the details of the constructions thanks to universality. Adding some matter fields or gauge fields [11] is another possibility to obtain a continuum theory, though it changes the theory to be constructed. We stick to pure gravity as the simplest case in this paper.

We perform a numerical simulation of this model using the multicanonical technique [12], which is powerful especially in the presence of large potential barriers between some local minima, as in the present case in which the system undergoes a first order phase transition. We measure the quantity which corresponds to the 'temperature' in the microcanonical ensemble, which enables us to extract various important thermodynamical properties of the model very clearly. We first clarify the first order nature of the phase transition with the Einstein-Hilbert action in several ways including a high precision finite size scaling analysis. We next add a new term to the action, which is motivated by a possible contribution from the measure for the path integral over the metric. The modified action has been first studied in four dimensions [13. Recently it has been studied in three dimensions by the Monte Carlo renormalization group and a possibility was suggested that the first order phase transition ceases to exist at a finite coefficient of the new term [14]. We confirm this possibility by investigating the expanded phase diagram 円.

The phase transition is expected to become second order at the end point of the phase transition line. We therefore attempt to observe the continuum physics at this end point. We measure the boundary area distribution, whose counterpart in $2 \mathrm{D}$ is known to show a clear scaling behavior [16, 17]. We indeed see a reasonable scaling behavior.

The paper is organized as follows. In Section 2, we explain the mul-

\footnotetext{
${ }^{1}$ While this paper was being completed, a preprint appeared [15], in which the authors address the same issue but with a conclusion which is different from ours.
} 
ticanoical techniques and introduce the quantity which corresponds to the 'temperature' in the microcanonical ensemble. In Section 3, we show the numerical results with the Einstein-Hilbert action, and clarify the first order nature of the phase transition. Above all, we observe a clear finite size scaling, which is consistent with the first order phase transition. In Section 4, we introduce the model with the new term. In Section 5, we show the results with the modified action and confirm that the first order phase transition line extends to the expanded phase diagram, ending at a point. In Section 6, we show the results for the boundary area distribution at the end point, which is consistent with fractal dimension three. Section $\square$ is devoted to conclusions and discussions.

\section{Multicanonical technique and "microcanon- ical inverse temperature"}

The naive discretization of the Einstein-Hilbert action in the dynamical triangulation model in three dimensions gives [1]

$$
S_{E H}=-\kappa_{0} N_{0}+\kappa_{3} N_{3},
$$

where $N_{0}$ and $N_{3}$ are the number of vertices and 3-simplices respectively in the simplicial manifold. $\kappa_{0}$ corresponds to the inverse of the gravitational constant, while $\kappa_{3}$ corresponds to the cosmological constant. Since we consider ensembles with fixed $N_{3}$, we use only the first term $S_{0}=-\kappa_{0} N_{0}$ in the following.

The partition function of the system with fixed $N_{3}$ is given as follows.

$$
\begin{aligned}
Z\left(\kappa_{0} ; N_{3}\right) & \equiv \sum_{\left\{T\left(N_{3}\right)\right\}} \exp \left(-S_{0}\right) \\
& =\sum_{N_{0}} P\left(N_{0}, \kappa_{0} ; N_{3}\right), \\
P\left(N_{0}, \kappa_{0} ; N_{3}\right) & \equiv n\left(N_{0} ; N_{3}\right) \exp \left(\kappa_{0} N_{0}\right) .
\end{aligned}
$$

The summation in the first line of (2) is taken over all possible triangulations of 3-dimensional simplicial manifolds with sphere topology that contain $N_{3}$ 3-simplices. $n\left(N_{0} ; N_{3}\right)$ is the number of the possible triangulations with a further restriction that they contain $N_{0}$ vertices. $P\left(N_{0}, \kappa_{0} ; N_{3}\right)$ represents the node number distribution for a canonical ensemble with fixed $\kappa_{0}$. In the 
following we suppress the argument $N_{3}$ in the $P\left(N_{0}, \kappa_{0} ; N_{3}\right)$ and $n\left(N_{0} ; N_{3}\right)$, unless necessary.

As in all of the recent numerical works on higher dimensional dynamical triangulation model, we use the so-called $(p, q)$ moves, which are proven to be ergodic [18], to simulate this system. Actually, $N_{3}$ is not kept fixed in the moves. We therefore have to allow a small fluctuation of $N_{3}$ around a target $N_{3}$ with an appropriate Gaussian potential for $N_{3}$. Measurement has been done only when the $N_{3}$ agrees with the target $N_{3}$. We refer to it as a simulation for fixed $N_{3}$ for simplicity.

The system is known to undergo a first order phase transition at some critical $\kappa_{0}$. Near the critical point, $P\left(N_{0}, \kappa_{0}\right)$ exhibits a double-peak structure. As one increases the system size $N_{3}$, the valley between the double peaks becomes deeper and deeper, which makes the ordinary canonical simulation increasingly difficult, due to the long Monte Carlo time required to travel back and forth between the two peaks for sufficiently many times. We perform the multicanonical simulation to overcome this problem. For technical details, we refer the readers to Ref. [19]. The simulation is performed by replacing the Boltzmann weight $\exp \left(\kappa_{0} N_{0}\right)$ with some function of $N_{0}$, which is determined iteratively so that the distribution of $N_{0}$ becomes as constant as possible within some window of $N_{0}$. If the window is wide enough to cover the whole support of the distribution function of $N_{0}$ for a canonical ensemble with some $\kappa_{0}$, one can reproduce the expectation values of any observables in the canonical ensemble by taking account of the effect of replacing the Boltzmann weight in updating the system. The idea is essentially a generalization of the histogram method. If one extends the window by fine-tuning the Boltzmann weight, the simulation includes information of canonical ensembles with various $\kappa_{0}$ in some finite region. This enables, for example, a precise identification of the peak of the node number susceptibility as a function of $\kappa_{0}$, which is essential in the high precision finite size scaling analysis.

Another advantage of the multicanonical simulation is that it allows us to extract the entropy as a function of $N_{0}$ defined by

$$
\mathcal{S}\left(N_{0}\right)=\log n\left(N_{0}\right),
$$

or its first derivative

$$
K\left(N_{0}\right)=-\left\{\mathcal{S}\left(N_{0}+1\right)-\mathcal{S}\left(N_{0}\right)\right\} .
$$


$K\left(N_{0}\right)$ is the analogue of the inverse temperature $\beta=1 / T$ defined in the microcanonical ensemble if we regard $-N_{0}$ as the energy. We therefore refer to $K\left(N_{0}\right)$ as the microcanonical inverse temperature. Strictly speaking, the simulation allows us to extract the entropy $\mathcal{S}\left(N_{0}\right)$ only up to an additional constant independent of $N_{0}$. Once $\mathcal{S}\left(N_{0}\right)$ is known, one can calculate the node number distribution $P\left(N_{0}, \kappa_{0}\right)$ in the canonical ensemble for arbitrary $\kappa_{0}$ through

$$
P\left(N_{0}, \kappa_{0}\right)=\exp \left\{\kappa_{0} N_{0}+\mathcal{S}\left(N_{0}\right)\right\}
$$

From this, we have

$$
\log P\left(N_{0}+1, \kappa_{0}\right)-\log P\left(N_{0}, \kappa_{0}\right)=\kappa_{0}-K\left(N_{0}\right)
$$

which means that if $\kappa_{0}-K\left(N_{0}\right)$ is positive (negative) for some $N_{0}$, the node number distribution $P\left(N_{0}, \kappa_{0}\right)$ increases (decreases) as $N_{0}$ increases. This is illustrated in Fig. 1. The upper part shows the microcanonical inverse temperature $K\left(N_{0}\right)$ as a function of $N_{0}$, with three horizontal lines $K=\kappa_{0}$ corresponding to $\kappa_{0}=\kappa_{0}^{s}, \kappa_{0}^{m}$ and $\kappa_{0}^{l}$. The lower part shows the node number distribution $P\left(N_{0}, \kappa_{0}\right)$ for each of the three values of $\kappa_{0}$. The intersections of the curve of the microcanonical inverse temperature and the horizontal lines give the extrema of the node number distribution for each $\kappa_{0}$.

When $\kappa_{0}=\kappa_{0}^{m}$, there are three intersection points: $\kappa_{0}^{m}=K\left(N_{0}^{(1)}\right)$, $K\left(N_{0}^{(2)}\right)$, and $K\left(N_{0}^{(3)}\right)$, where $N_{0}^{(1)}<N_{0}^{(2)}<N_{0}^{(3)}$. The node number distribution has the double peaks at $N_{0}^{(1)}$ and $N_{0}^{(3)} \cdot N_{0}^{(2)}$ gives the place of the local minimum. From (7), the ratios $P\left(N_{0}^{(1)}, \kappa_{0}^{m}\right) / P\left(N_{0}^{(2)}, \kappa_{0}^{m}\right)$ and $P\left(N_{0}^{(3)}, \kappa_{0}^{m}\right) / P\left(N_{0}^{(2)}, \kappa_{0}^{m}\right)$ are given by the exponential of the area of the light and heavy shaded regions in Fig. 1, respectively. Since we have chosen $\kappa_{0}^{m}$ so that the area of the two regions is equal, the peaks of the node number distribution have the same height.

In general, when the microcanonical inverse temperature has a local minimum and a local maximum, the node number distribution shows a double peak structure for the canonical ensemble with $\kappa_{0}$ set to the value between the local minimum and the local maximum. The quantity, therefore, serves as a useful probe to clarify the fate of the first order phase transition when one modifies the action from the Einstein-Hilbert action. 


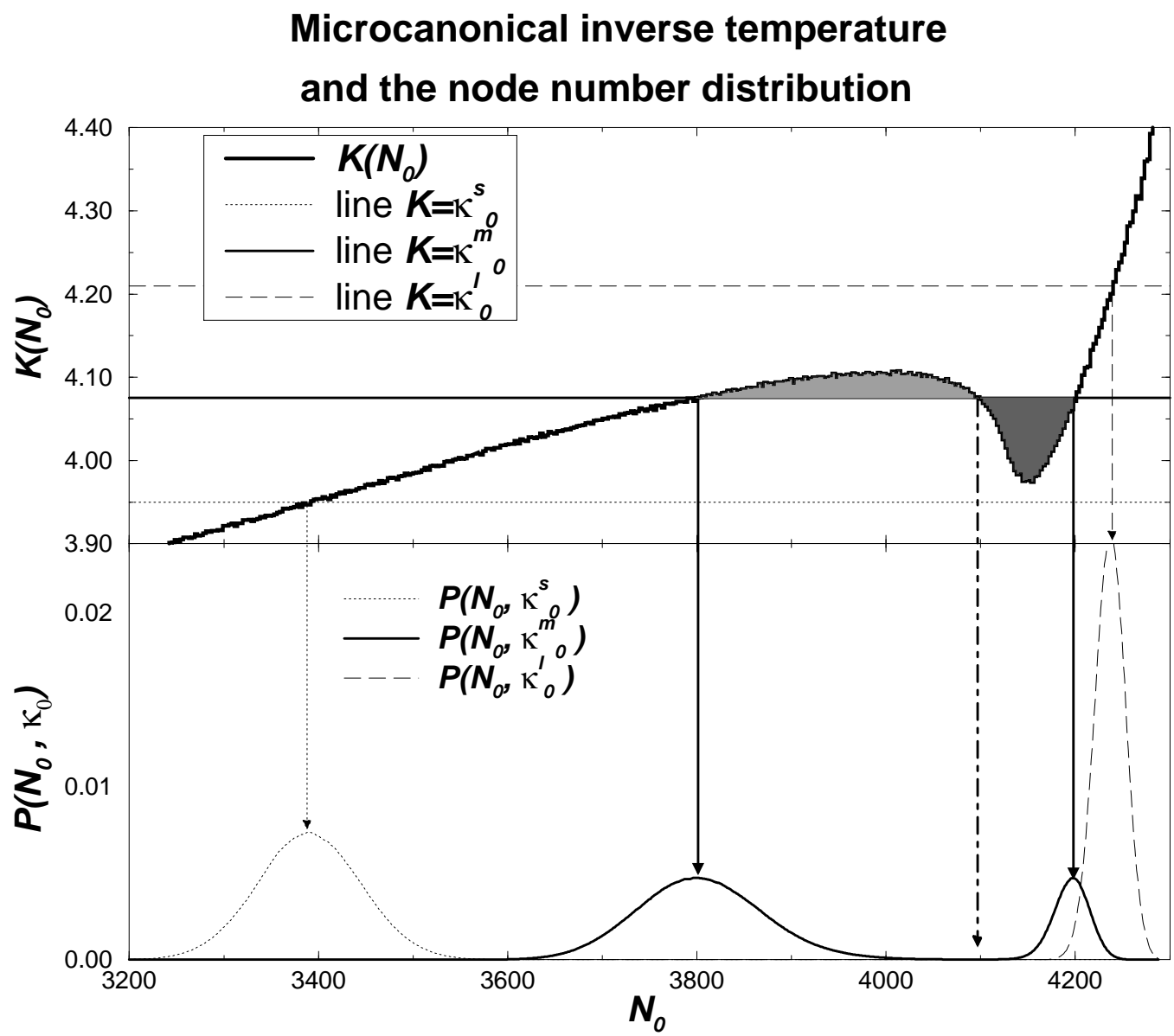

Figure 1: The upper part shows the microcanonical inverse temperature $K\left(N_{0}\right)$ (heavy solid curve) with three horizontal lines $K=\kappa_{0}$ corresponding to $\kappa_{0}=\kappa_{0}^{s}, \kappa_{0}^{m}$ and $\kappa_{0}^{l}$. The lower part shows the node number distribution $P\left(N_{0}, \kappa_{0}\right)$ for each of the three values of $\kappa_{0}$. 


\section{microcanonical inverse temperature $K$ vs $N_{0} / N_{3}$}

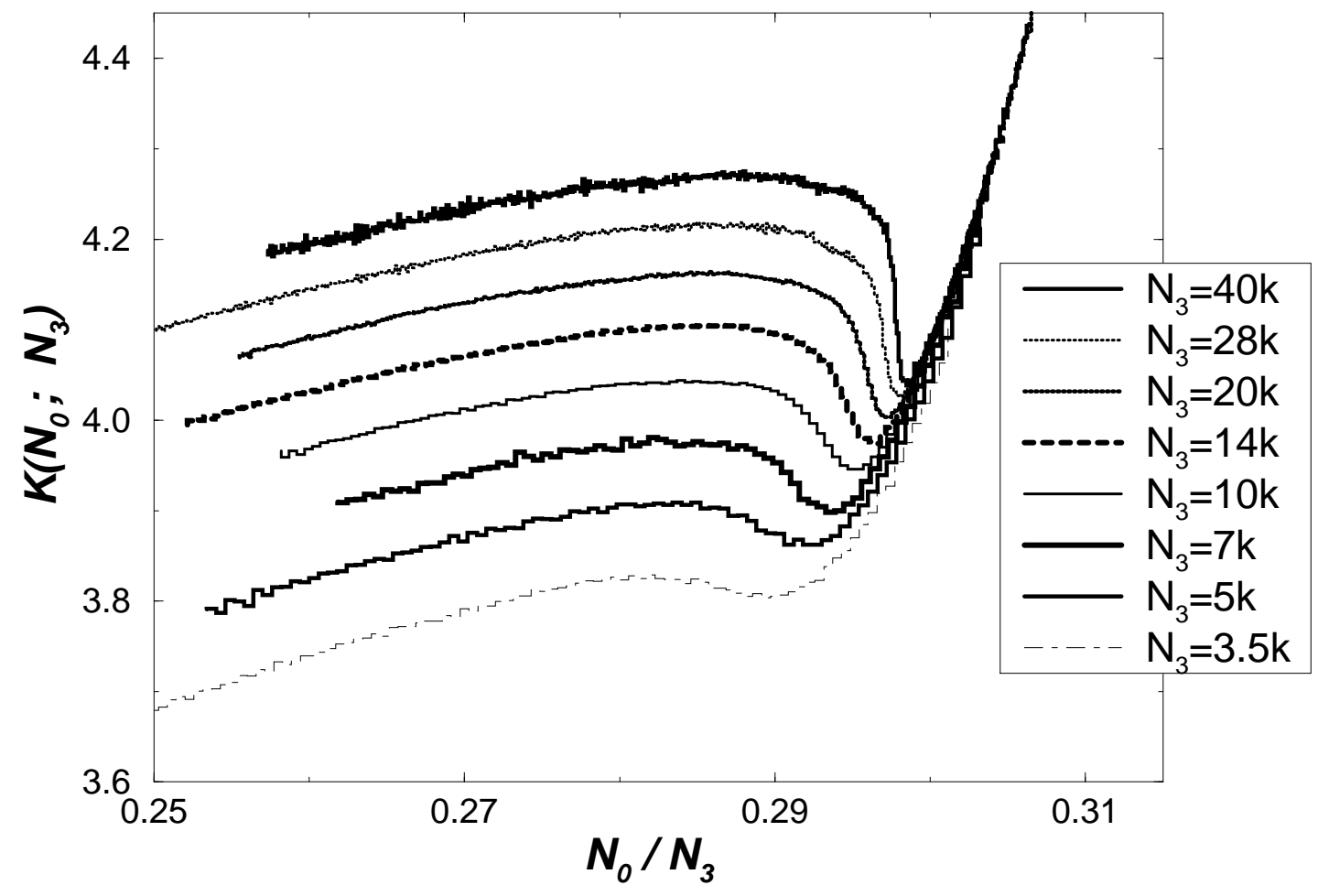

Figure 2: The microcanonical inverse temperature $K\left(N_{0} ; N_{3}\right)$ is plotted against the node number $N_{0}$ normalized by the system size $N_{3}$ for $N_{3}=3,500$ to 40,000 .

\section{The results for the Einstein-Hilbert action}

In this section, we show the results for the Einstein-Hilbert action and clarify the first order nature of the phase transition by the measurement of various thermodynamical quantities.

We first show the microcanonical inverse temperature $K\left(N_{0} ; N_{3}\right)$ for various system size from $N_{3}=3,500$ to 40,000 in Fig. 2. Since the microcanonical inverse temperature has a local minimum and a local maximum, the node number distribution shows a double peak structure at some region of $\kappa_{0}$. We 


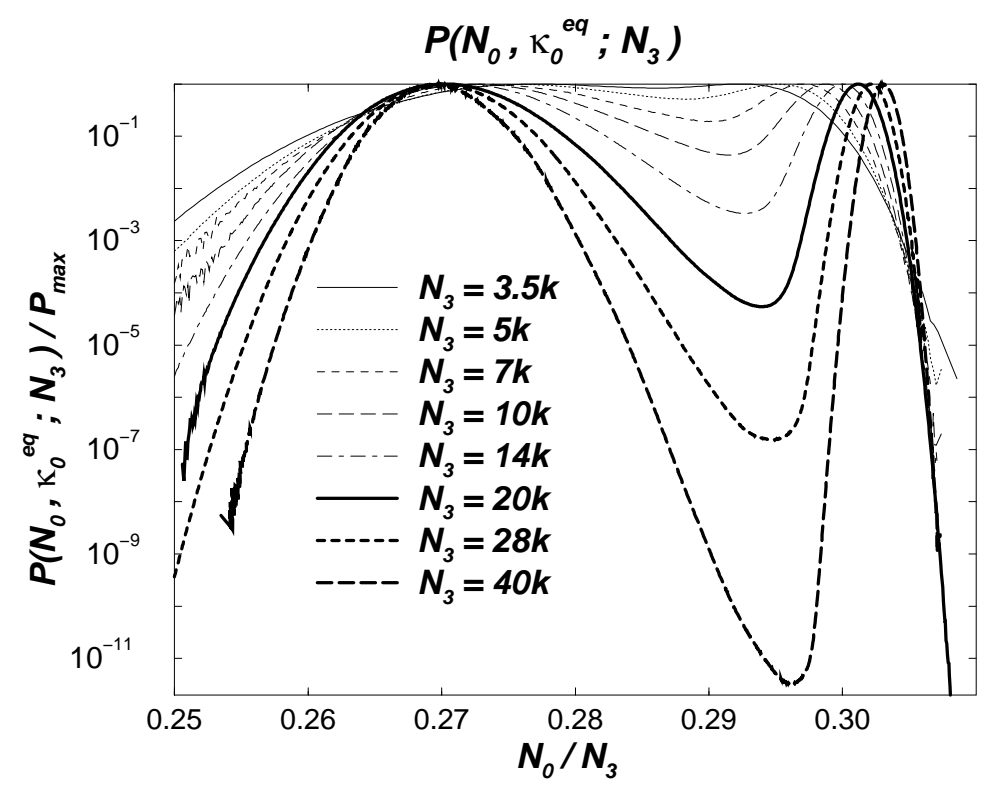

Figure 3: The node number distribution at $\kappa_{0}=\kappa_{0}^{e q}$ normalized by the peak height, $P_{\max }$, is plotted against $N_{0} / N_{3}$ for $N_{3}=3.5 \mathrm{k}, \cdots 40 \mathrm{k}$.

choose the $\kappa_{0}$ with which the height of the peaks is the same. We denote such $\kappa_{0}$ by $\kappa_{0}^{e q}$. The determination of $\kappa_{0}^{e q}$ can be done easily by referring to the microcanonical inverse temperature as is explained in the previous section. Fig. 3 shows the results for the node number distribution at $\kappa_{0}^{e q}$ with the system size from $N_{3}=3,500$ to 40,000 . The logarithm of the ratio of the height of the peaks to that of the valley gives the interfacial energy. (See Ref. [19] for further analysis on this quantity.) Note that the ratio is about $10^{12}$ for $N_{3}=40,000$. One can easily understand that ordinary canonical simulations cannot be used in this situation.

In Fig. 4, we plot the mean node number in the canonical ensemble with $\kappa_{0}$ against $\kappa_{0}$. We replot the microcanonical inverse temperature with $\kappa_{0}=K\left(N_{0} ; N_{3}\right)$ for comparison. The mean node number shows a sharp increase in the critical region of $\kappa_{0}$ for a sufficiently large system size, which is another way of observing the signal of the first order phase transition. The data curve of the mean node number coincides with the data curve of the microcanonical inverse temperature except in the critical region, as is expected. 


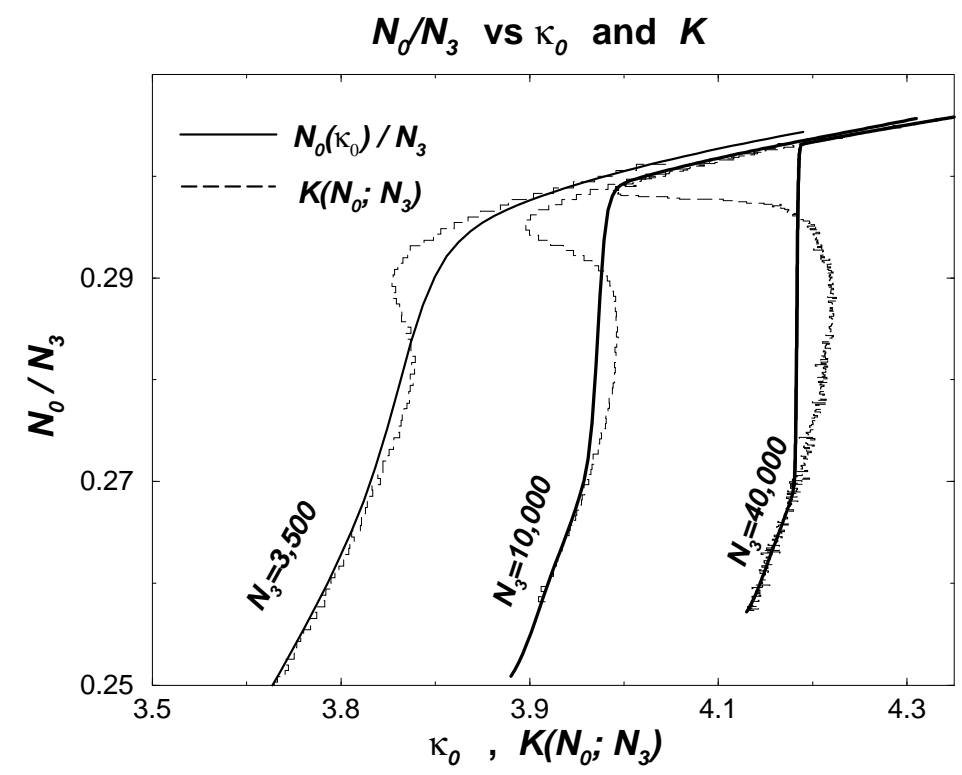

Figure 4: The mean node number normalized by the system size $N_{3}$ is plotted against $\kappa_{0}$ by the solid line. The microcanonical inverse temperature is replotted by the dashed line for comparison. 


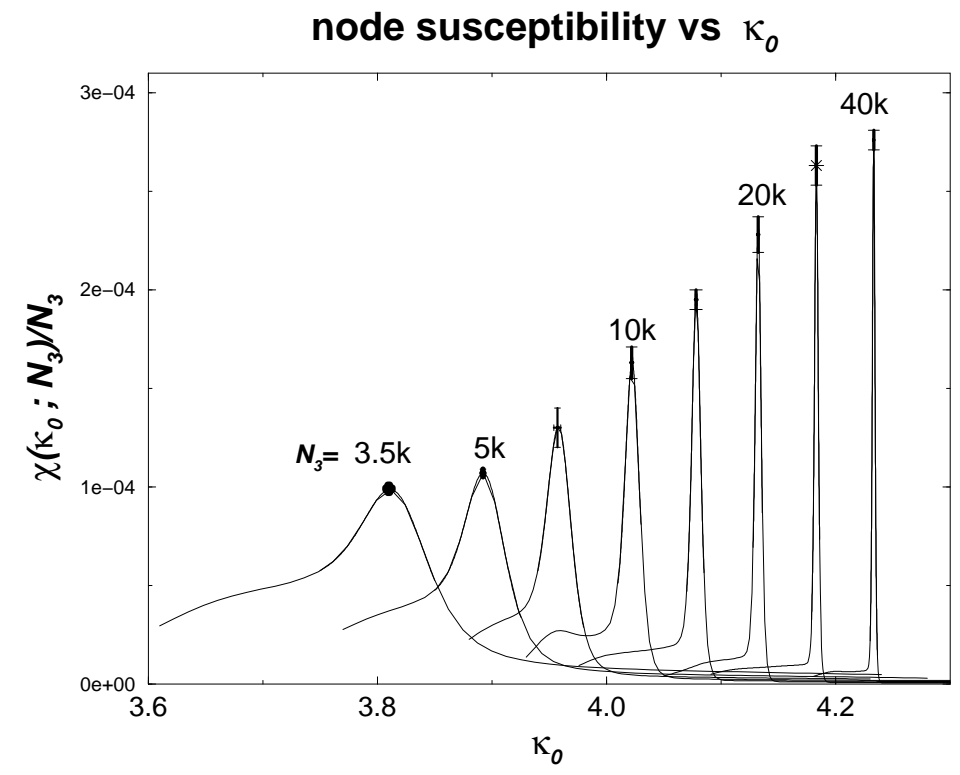

Figure 5: The node number susceptibility $\chi\left(\kappa_{0} ; N_{3}\right)$ is plotted against $\kappa_{0}$ for the system size from $N_{3}=3,500$ to 40,000 .

In Fig. 5 we show the result for the node number susceptibility $\chi\left(\kappa_{0} ; N_{3}\right) \equiv$ $\left(\left\langle N_{0}^{2}\right\rangle-\left\langle N_{0}\right\rangle^{2}\right) / N_{3}$. We call the $\kappa_{0}$ which gives the peak of the susceptibility for each $N_{3}$ as the pseudo-critical point $\kappa_{0}^{c}\left(N_{3}\right)$. In ordinary statistical systems, the peak height of the susceptibility grows as a power of the system size. If the peak is due to a first order phase transition, the internal energy distribution shows a double peak structure as the one in Fig. 3, and the tunneling between the two peaks causes a linear growth of the peak height of the susceptibility. In Fig. 6 we plot the peak height of the susceptibility against the system size. The data for the four large $N_{3}$ 's can be fitted very nicely to the formula

$$
\chi\left(\kappa_{0}^{c}\left(N_{3}\right) ; N_{3}\right)=p_{1}+p_{2} N_{3},
$$

with $p_{1}=-1.8(2)$ and $p_{2}=3.20(7) \times 10^{-3}$, which is consistent with the finite size scaling for the first order phase transition.

The pseudo-critical coupling constant $\kappa_{0}^{c}\left(N_{3}\right)$ shifts to the right with no tendency of convergence as one increases the system size up to $N_{3}=40,000$. This behavior should be contrasted with ordinary statistical systems, for 


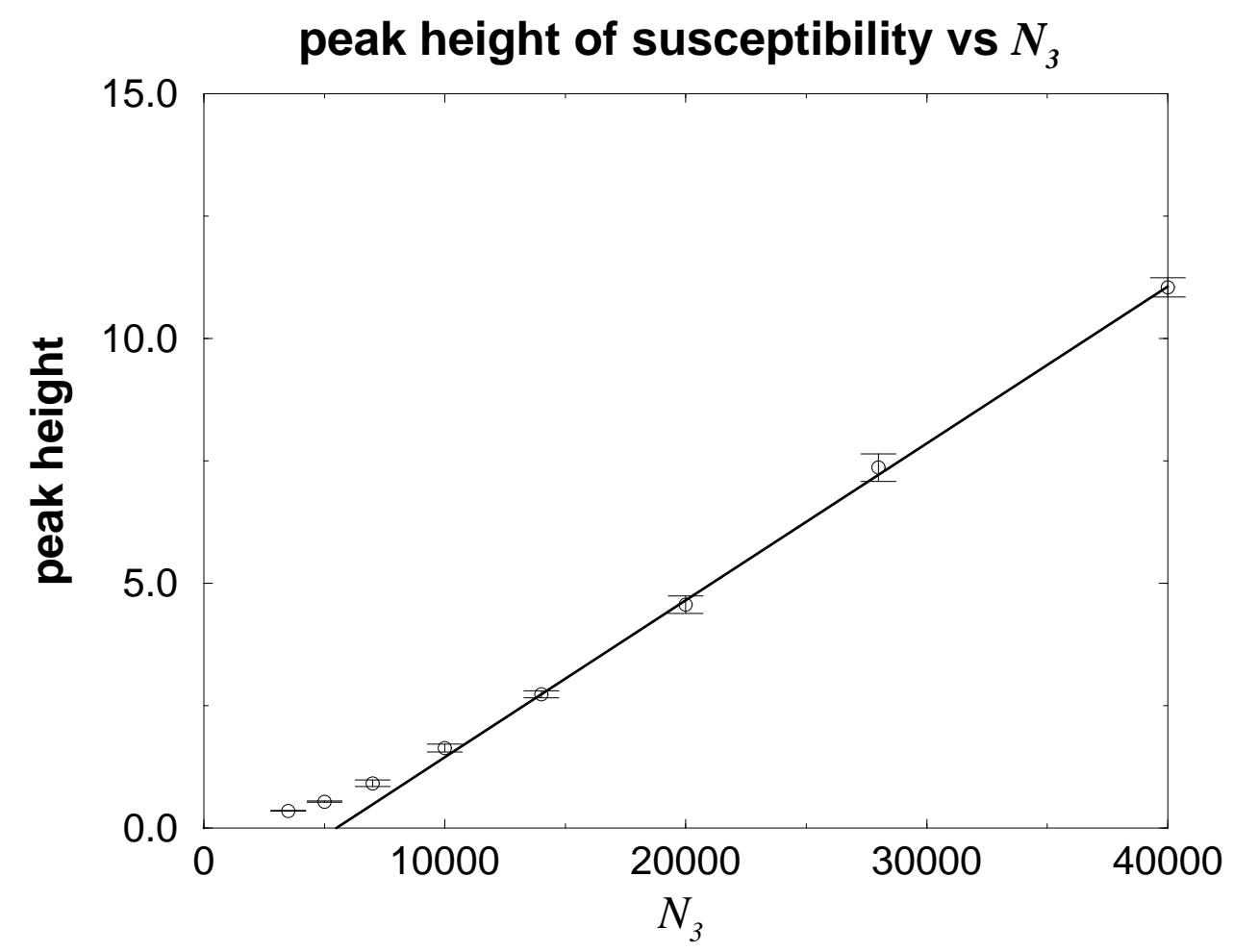

Figure 6: The peak height of the susceptibility is plotted against $N_{3}$. The straight line show the linear fit $\chi / N_{3}=p_{1}+p_{2} N_{3}$, which is consistent with the first order phase transition. The $\chi^{2}$ of the fit is $\chi^{2} / d o f=0.3$. We use only the data for the four large $N_{3}$ 's. 
which the dependence of the pseudo-critical coupling on the system size $V$ obeys the scaling relation such as

$$
\kappa_{0}^{c}(V)=\kappa_{0}^{c}(\infty)+c V^{-p}
$$

The unusual shift of the pseudo-critical coupling constant has been discussed earlier in the four-dimensional dynamical triangulation model [20].

\section{The modified action}

In this section, we modify the Einstein-Hilbert action (11) and attempt a search for a second order phase transition point in an expanded phase diagram. We consider the following action.

$$
\begin{aligned}
S & =S_{0}-\mu M, \\
M & =\sum_{v} \log [o(v) / 4],
\end{aligned}
$$

where $\mu$ is the new coupling constant 2 , and $o(v)$ is the number of 3 -simplices that contain the node $v$. The summation in (10) is taken over all the nodes in the manifold. This term [13] can be considered as a possible contribution from the path integral measure $\Pi_{x} g(x)^{\mu / 2}$, since $o(v)$ can be interpreted as the "local volume", which corresponds to $\sqrt{g(x)}$ in the continuum.

In the following we consider the canonical ensemble with fixed $N_{3}$ given by the partition function :

$$
\begin{aligned}
Z^{(\bmod )}\left(\kappa_{0} ; \mu ; N_{3}\right) & =\sum_{\left\{T\left(N_{3}\right)\right\}} \exp \left(\kappa_{0} N_{0}+\mu M\right) \\
& =\sum_{N_{0}} z\left(N_{0} ; \mu ; N_{3}\right) \exp \left(\kappa_{0} N_{0}\right)
\end{aligned}
$$

where $z\left(N_{0} ; \mu ; N_{3}\right)$ is the partition function for fixed $N_{0}$, which can be given by

$$
z\left(N_{0} ; \mu ; N_{3}\right)=\sum_{\left\{T\left(N_{3}, N_{0}\right)\right\}} \exp (\mu M)
$$

\footnotetext{
${ }^{2}$ Note that the $\mu$ defined here is the same as the one in Refs. [14, 15]. The definition of $\mu$ used in Ref. 21 corresponds to $-\mu$ in this paper.
} 
where the summation is now taken over all possible triangulations that contain $N_{3} 3$-simplices and $N_{0}$ vertices. As before, we suppress the argument $N_{3}$ in $z\left(N_{0} ; \mu ; N_{3}\right)$ unless necessary. In this paper, we concentrate on the negative $\mu$ region, in which the first order phase transition line is predicted to end at a point through the MCRG analysis in Ref. [14].

\section{End point of the first order phase transi- tion}

Let us define the (generalized) microcanonical entropy

$$
\mathcal{S}\left(N_{0} ; \mu\right) \equiv \log z\left(N_{0} ; \mu\right)
$$

and its first derivative

$$
K\left(N_{0} ; \mu\right) \equiv-\left\{\mathcal{S}\left(N_{0}+1 ; \mu\right)-\mathcal{S}\left(N_{0} ; \mu\right)\right\}
$$

which we keep on referring to as the microcanonical inverse temperature.

In Fig. 7, we show the microcanonical inverse temperature $K\left(N_{0} ; \mu\right)$ for several negative values of $\mu$. As was explained in Section 2, the double peak structure in the node number distribution, which is a signal of the first order phase transition, can be probed directly by looking at the microcanonical inverse temperature $K\left(N_{0} ; \mu\right)$.

One can see that the local minimum and the local maximum observed for $\mu=0$ merge at $\mu^{*}=-0.65$, beyond which the $K\left(N_{0} ; \mu\right)$ becomes a monotonous function of $N_{0}$. This suggests that the first order phase transition ceases to exist at $\mu^{*}$, beyond which it becomes a crossover.

For each $\mu \geq \mu^{*}$, we define the pseudo-critical coupling $\kappa_{0}^{c}$ as the position of the peak of the node number susceptibility $\chi_{0}\left(\kappa_{0}, \mu\right)$. The end point of the phase transition line is given by $\mu^{*}=-0.65, \kappa_{0}^{c}=5.00$ for $N_{3}=10,000$, and $\mu^{*}=-0.80, \kappa_{0}^{c}=5.515$ for $N_{3}=20,000$. In Fig. 8, we plot the free energy $f\left(N_{0} ; \mu ; \kappa_{0}\right)=-\mathcal{S}\left(N_{0} ; \mu\right)-\kappa_{0} N_{0}$ as a function of $N_{0}$ near $\kappa_{0}^{c}$ for $\mu=0$ and $\mu=\mu^{*}$. The two local minima observed for $\mu=0$ merge into one at $\mu=\mu^{*}$. The free energy curve at $\left(\kappa_{0}^{c}, \mu^{*}\right)$ has a wide flat bottom, which suggests the existence of a massless mode. 


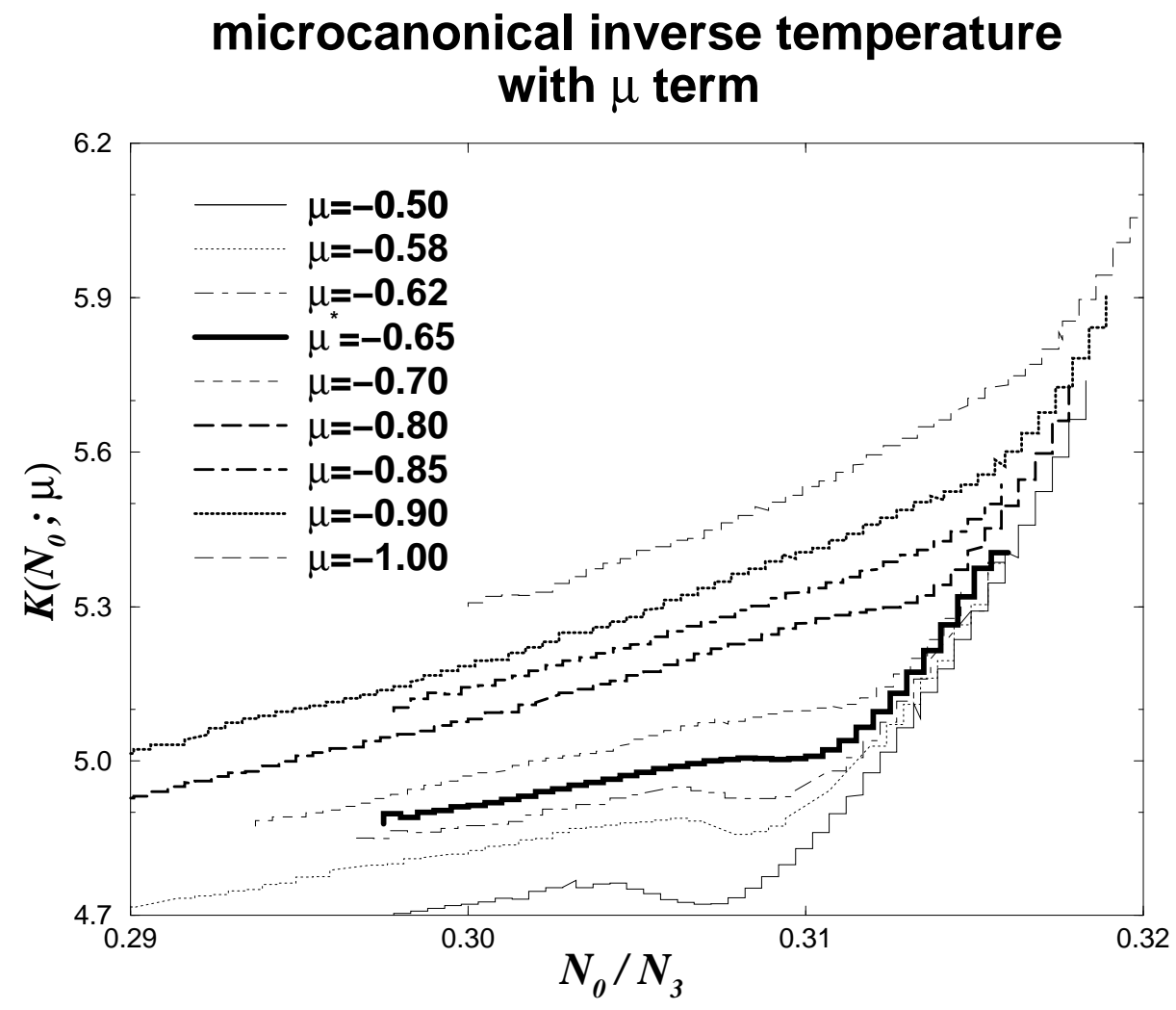

Figure 7: The microcanonical inverse temperature $K\left(N_{0} ; \mu\right)$ as a function of $N_{0}$ for several $\mu$ 's with the system size $N_{3}=10,000$. 


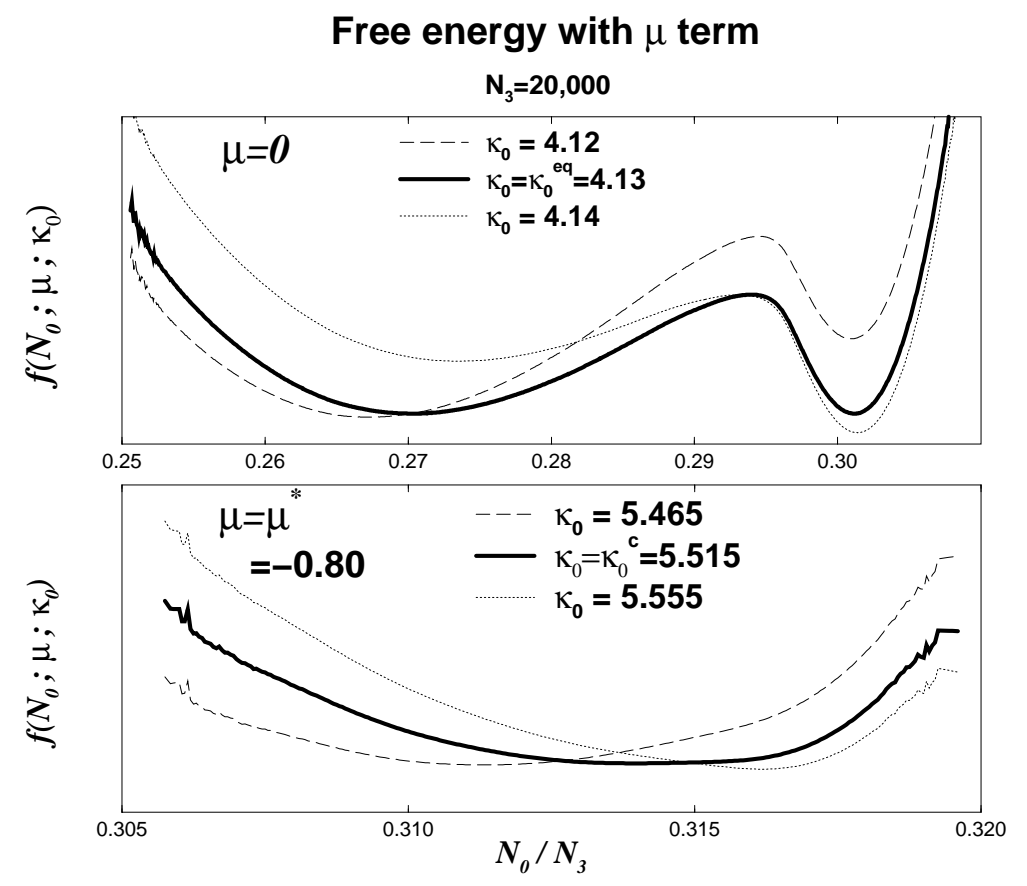

Figure 8: The free energy $f\left(N_{0} ; \mu, \kappa_{0}\right)$ as a function of $N_{0}$ near $\kappa_{0}^{c}$ for $\mu=0$ and $\mu=\mu^{*}=-0.80$ with the system size $N_{3}=20,000$.

\section{$6 \quad$ Fractal structure at the end point}

In order to examine a possible continuum limit at the end point, we measure the boundary area distribution $\rho(A, r)$, which is the number of boundaries with the area $A$ at the geodesic distance $r$. The corresponding quantity in two dimensions called the loop length distribution is calculated analytically and is found to possess a continuum limit [16]. The scaling behavior expected from this result has been correctly reproduced by a numerical simulation [17.

In Fig. 9, we plot the boundary area distribution at the end point of the first order phase transition line as a function of $x=A / r^{2}$. One can see a reasonable scaling behavior. The power of $r$ in the scaling variable $x=A / r^{2}$

implies that the fractal dimension is 3 . We would like to remark that the scaling behavior we observe is much better than the one that has been claimed to exist with the unmodified action [22]. 


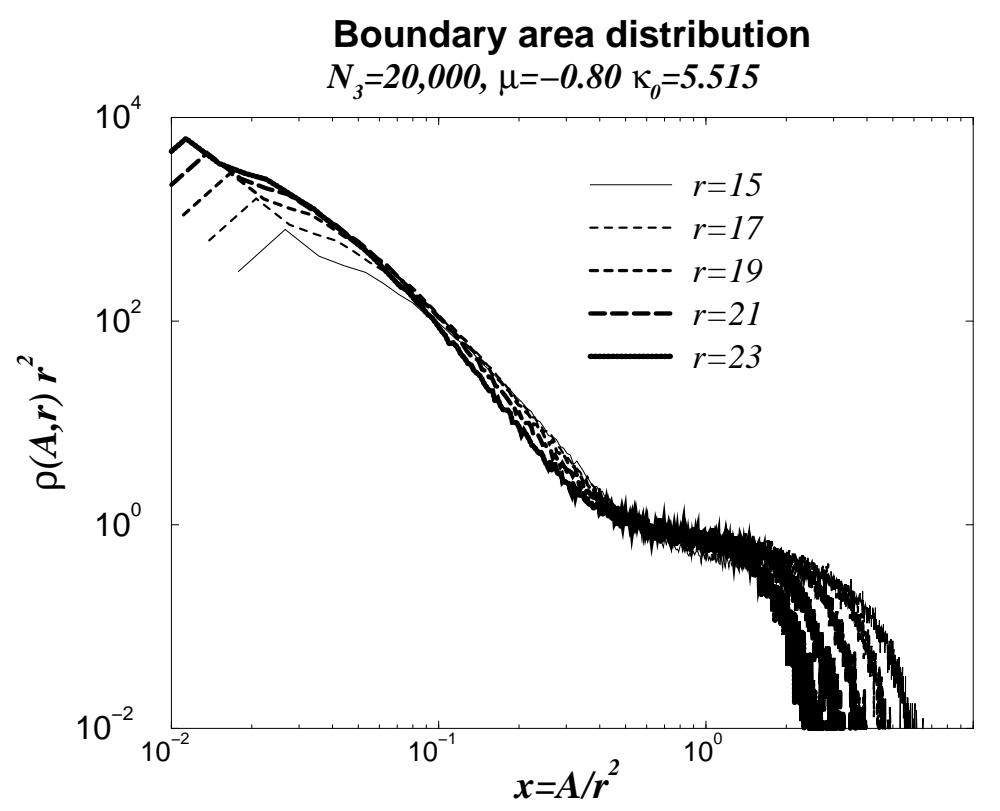

Figure 9: Boundary area distribution $\rho(A, r)$ at the end point of the first order phase transition line with the system size $N_{3}=20,000$. 


\section{Conclusions and discussions}

We applied the multicanonical technique to the three dimensional dynamical triangulation model. The microcanonical inverse temperature served as a useful tool to clarify the first order nature of the phase transition observed for the Einstein-Hilbert action and to investigate its fate in the expanded phase diagram with a new coupling constant.

Our results about the modified action suggest that the first order phase transition becomes second order at the end point, where one can construct a continuum theory. A natural question to be asked is what the continuum theory can be. One may suspect that we cannot construct any continuum theory in three-dimensional quantum gravity, since we have no physical degrees of freedom. This argument is too naive, however. An example of field theory which has no physical degrees of freedom that is still completely well-defined is two-dimensional Yang-Mills theory.

The fact that we have to fine-tune two parameters to obtain the continuum limit implies that there are two relevant operators around the fixed point. A possible interpretation of the continuum theory is therefore the $R^{2}$ gravity. The observation that the fractal dimension at the fixed point is approximately three is also suggestive of this interpretation. The fractal dimension we extracted, however, is still preliminary, and the real value, which could be obtained by increasing the system size might well be larger.

In Ref. [15, the authors pointed out that the system in the negative $\mu$ region might be suffered with large finite size effects. Taking this into account, they claimed that the line of first order phase transition continues to $\mu=\mu^{c}$ and $\kappa_{0}=\infty$ and that the system is in the crumpled phase for all $\kappa_{0}$ when $\mu<\mu^{c}$. Needless to say, simulations with larger system size are necessary to discriminate between these possibilities.

In Ref. [7], we examined a different modified action in four-dimensional dynamical triangulation model. The added term was

$$
S_{u}=u \sum_{v}[o(v)-5]^{2} .
$$

We saw that the phase transition turned into a cross over even for a small positive $u$ and the system was observed to be always in the branched polymer phase. Let us write the term to be added as $\sum_{v} f(o(v))$. Note that a constant term and a linear term in $f(x)$ can be absorbed by the redefinition of $\kappa_{0}$ and

$\kappa_{3}$. The quadratic term in (16) might be too strong since it dominates the behavior of $f(x)$ in the large $x$ region. 
In Ref. [15], another possibility has been suggested that the line of the first order phase transition ends at a point with positive $\mu$. It is of interest to clarify this possibility as well using the technique we used, although the simulation must be much harder due to the small acceptance rate [15].

\section{Acknowledgments}

We would like to thank A. Fujitsu for helpful discussions and comments. Most of the calculations in this paper has been done on work stations in Aizu University.

\section{References}

[1] E. Brézin, C. Itzykson, G. Parisi and J.-B. Zuber, Commun. Math. Phys. 59 (1978) 35; F. David, Nucl. Phys. B257 (1985) 45; V.A. Kazakov, Phys. Lett. 150B (1985) 282; D.V. Boulatov, V.A. Kazakov, I.K. Kostov and A.A. Migdal, Nucl. Phys. B275 (1986) 641; J. Ambjørn, B. Durhuus and J. Fröhlich, Nucl. Phys. B257 (1985) 433.

[2] A.M. Polyakov, Mod. Phys. Lett. A2 (1987) 893; V.G. Knizhnik, A.M. Polyakov and A.B. Zamolodchikov, Mod. Phys. Lett. A3 (1988) 819; F. David, Mod. Phys. Lett. A3 (1988) 1651; J. Distler and H. Kawai, Nucl. Phys. B321 (1989) 504.

[3] C. Holm and W. Janke, Phys. Lett. B335 (1994)143; J. Nishimura and M. Oshikawa, Phys. Lett. B338 (1994) 187; W. Bock and J.C. Vink, Nucl. Phys. B438 (1995) 320; C. Holm and W. Janke, Nucl. Phys. B477 (1996) 465; J. Nishimura, Prog. Theor. Phys. 94 (1995) 299.

[4] M. Agishtein and A. Migdal, Mod. Phys. Lett. A6 (1991) 1963; J. Ambjorn, D.V. Boulatov, A. Krzywicki and S. Varsted, Phys. Lett. B276 (1992) 432 .

[5] P. Bialas, Z. Burda, A. Krzywicki and B. Petersson, Nucl. Phys. B472 (1996) 293.

[6] B. de Bakker, Phys. Lett. B389 (1996) 238; S. Catteral, R. Renken and J. Kogut, hep-lat/9709007. 
[7] T. Hotta, T. Izubuchi and J. Nishimura, Prog. Theor. Phys. 94 (1995) 263.

[8] S. Catterall, G. Thorleifsson and J. Kogut, Nucl. Phys. B468 (1996) 263.

[9] T. Aida, Y. Kitazawa, H. Kawai and M. Ninomiya, Nucl. Phys. B427 (1994) 158; T. Aida, Y. Kitazawa, J. Nishimura and A. Tsuchiya, Nucl. Phys. B444 (1995) 353.

[10] E. Witten, Nucl. Phys. B460 (1996) 335.

[11] S. Bilke, Z. Burda, A. Krzywicki, B. Petersson, J. Tabaczek and G. Thorleiffson, hep-lat/9710077.

[12] See B.A. Berg, hep-lat/9708003, to appear in Nucl. Phys. Proc. Suppl. $\mathbf{B}$, and the references therein.

[13] B. Brugmann and E. Marinari, Phys. Lett. B349 (1995) 35.

[14] R. L. Renken, Nucl. Phys. B485 (1997) 503.

[15] R.L. Renken, S.M. Catterall and J.B. Kogut, hep-lat/9712011.

[16] H. Kawai, N. Kawamoto, T. Mogami and Y. Watabiki, Phys. Lett. B306 (1993) 19.

[17] N. Tsuda and T. Yukawa, Phys. Lett. B305 (1993) 223.

[18] M. Gross and S. Varsted, Nucl. Phys. B378 (1992) 367.

[19] A. Fujitsu and T. Izubuchi, in preparation.

[20] B.V. de Bakker and J. Smit, Phys. Lett. B334 (1994) 304.

[21] T. Hotta, T. Izubuchi and J. Nishimura, hep-lat/9710017.

[22] H. Hagura, N. Tsuda and T. Yukawa, hep-lat/9512016. 